\title{
Experimental investigation of the vibrational density of states and electronic excitations in metallic nanocrystals
}

\author{
M. Bayle, P. Benzo, N. Combe, C. Gatel, C. Bonafos, G. Benassayag, and R. Carles* \\ CEMES-CNRS Université de Toulouse 29 rue Jeanne Marvig, BP 94347, 31055 Toulouse Cedex 4, France
}

(Received 10 January 2014; revised manuscript received 16 April 2014; published 5 May 2014)

\begin{abstract}
An investigation of the vibrational density of states (VDOS) in silver nanocrystals is performed using Raman scattering. A specific sample architecture, setup configuration, and original elaboration process are used in order to take simultaneously advantage of spectrally and spatially localized surface plasmon resonance, optical amplification, and dark-field spectroscopy. Disentangling the contributions of atom vibrations and electron-hole excitations (i.e., the so-called "background" in surface-enhanced Raman scattering) is performed. The extracted VDOS is successfully compared with theoretical ones obtained by atomic scale simulations. The effects of size, strain, and disorder on the VDOS are analyzed; in particular, the strain effect is investigated experimentally using the geometrical phase analysis coupled with high-resolution transmission electron microscopy. This work offers an opportunity to examine thermodynamic properties, like specific heat, at the nanoscale.
\end{abstract}

DOI: 10.1103/PhysRevB.89.195402

PACS number(s): 63.22.Kn, 78.30.Er, 73.22.Lp, 65.80.-g

\section{INTRODUCTION}

Studies on metallic nanocrystals (NCs) are the subject of numerous works, either experimental or theoretical, due to their huge collective electronic response to light excitation in the visible or infrared range [1]. The localized surface plasmon resonance (LSPR) is widely exploited for enhanced optical spectroscopies of molecules [2], photothermal therapy [3], photovoltaics [4,5], or more recently in plasmoelectronics [6,7] and photocatalysis [8-10]. The photon-plasmon excitations are highly damped: relaxation of collective electronic excitations occurs in the femtosecond regime [11] through electron-electron interactions (the so-called Landau damping) whereas in the picosecond regime energy relaxation processes are mainly governed by electron-phonon interactions [12]. Acousticlike vibrations modulate in the $\mathrm{THz}$ range the polarizability of the confined electron gas and are easily detected in Raman-Brillouin scattering [13-15] or pumpprobe experiments $[11,12,16,17]$. The most relevant points concerning phonon properties and electron-phonon coupling are as follows:

(i) The phonon wave vector $\mathbf{k}$ is no longer a good "quantum" number to describe vibrations in NCs. Due to confinement, the vibrational modes are discretized inducing an acoustic gap. According to Lamb's approach [18], within the linear elasticity approximation and assimiling the $\mathrm{NC}$ to a homogeneous isotropic elastic sphere, the vibrational modes are categorized by their angular momentum $(\ell=0,1,2, \ldots)$ and harmonic $(n=1,2,3, \ldots)$ numbers.

(ii) Surface atoms play a significant role compared to inner atoms questioning then the validity of elastic approximation and interatomic spacing constancy.

(iii) New coupling channels appear for the interactions between photons and electrons (absorption, elastic scattering), and between electrons and phonons (inelastic scattering) because of the breakdown of $\mathbf{k}$-vector selection rules.

Most of the descriptions of electron-phonon couplings rely on phenomenological models where the vibrational states are

*robert.carles@cemes.fr described using a three-dimensional Debye model and electronic excitations as collective modes or plasmons $[12,16,17]$. However, more accurate descriptions of the vibrational density of states (VDOS) are required for describing thermodynamic and transport properties (specific heat, vibrational entropy, thermal and electric conductivity) [19-21]. Indeed in noble metals like silver, the Debye temperature is as low as $215 \mathrm{~K}$ [22], and as a consequence all the electronic excitations can potentially interact with the whole bath of phonons, at room temperature. The most accurate determinations of VDOS in noble metals can be only achieved through atomic simulations [15,17,23-27], because the linear elasticity approach is not reliable in the high-frequency (i.e., the short wavelength) range. However three main problems are not completely solved:

(i) In NCs, the VDOS is sensitive to surface and morphology [23]. Moreover strain field heterogeneity due to capillary pressure at the surface can also play a role [19,28,29].

(ii) For an embedded NC the surrounding matrix is generally not taken into account while its vibrational spectrum may overlap with the embedded NC one [30].

(iii) Contradictory results concern on one hand the discrepancy with the Debye model at low frequency (squared or linear frequency dependence) and on the other hand the origin of a high-frequency tail beyond the cutoff of the bulk material (internal strain effect or localized modes) $[21,26,31,32]$. These "anomalies" significantly contribute to the specific heat and to the vibrational entropy.

Few experimental techniques are available for exploring the whole phonon spectrum. Scarce and poorly resolved results have been obtained using inelastic neutron scattering, nuclear resonant inelastic x-ray scattering [20,32,33], or Raman scattering [34] on deposits of Pd and $\mathrm{Fe}$ nanograins or microcrystalline $\mathrm{Ag}$ or $\mathrm{Cu}$, respectively. At the moment, exploring the entire VDOS of nanostructures always remains a challenge due to the small scattering volume, low cross section for inelastic processes, and instability of NCs under intense beams. In this work, an experimental way to get accurate information on the VDOS of silver nanoparticles using Raman scattering is presented. The experimental result is then compared to atomistic calculations. Section II details the 
silver NCs preparation, their characteristics, and the Raman experimental setup. Section III reports the measured Raman spectra of the embedded NCs and its relation with the VDOS of the silver nanoparticles. Finally, Sec. IV compares the experimental VDOS deduced from the Raman spectra to the theoretical one. Strain effects induced by the capillary pressure are discussed, as well as the thermodynamic properties of the NC.

\section{EXPERIMENTAL METHOD}

\section{A. Silver NCs preparation}

We recently developed a synthesis technique based on implantation of $\mathrm{Ag}^{+}$ions with low kinetic energy (few $\mathrm{keV}$ ) and doses of few $10^{15}$ ions $/ \mathrm{cm}^{2}$ by which a single plane of silver nanocrystals (Ag NCs) is embedded near the free surface of $\mathrm{a} \mathrm{SiO}_{2}$ layer thermally grown on a $\mathrm{Si}$ wafer [35]. In Fig. 1(a) a typical transmission electron microscopy (TEM) image of a cross section on an implanted sample reveals the presence of $\mathrm{Ag} \mathrm{NCs}$ in a single plane located a few $\mathrm{nm}$ under the surface. These particles are formed during the implantation process because of the high mobility of $\mathrm{Ag}$ in $\mathrm{SiO}_{2}$; they are crystalline and made of pure silver, as revealed by their diffraction pattern [35]. They are spherical and homogeneously distributed as observed in the TEM plan view in Fig. 1(b). The corresponding size histogram is reported in Fig. 1(c): the distribution function is well described by a Gaussian with an average diameter $D \approx 4.6 \mathrm{~nm}$ and a standard deviation $\sigma \approx 1.0 \mathrm{~nm}$. The sizes are sufficiently smaller than their mutual distance (mean value $\approx 10 \mathrm{~nm}$ ) so that the vibrational or electronic mutual interactions can be neglected.
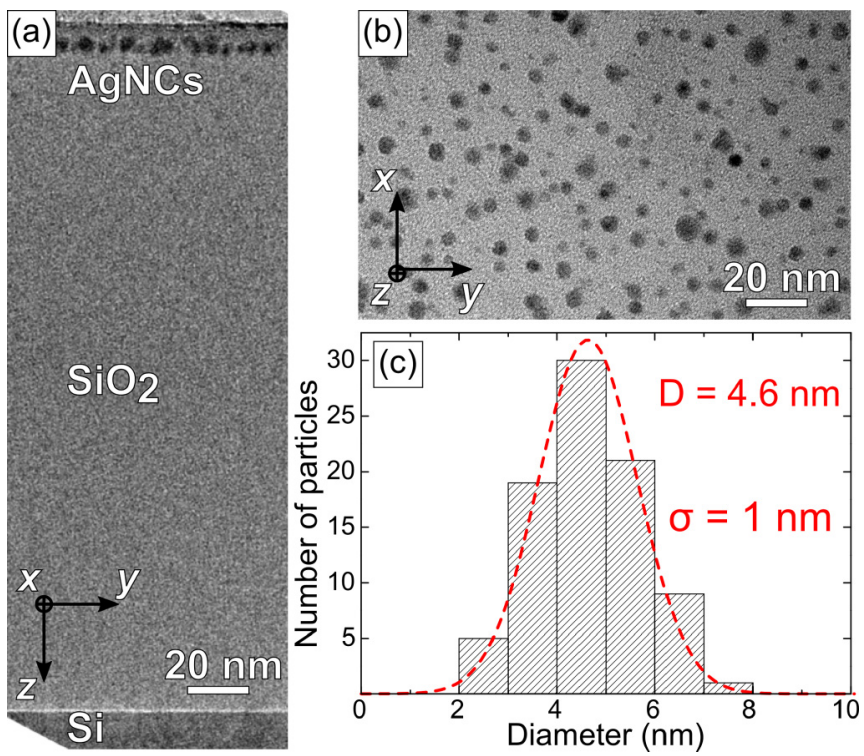

FIG. 1. (Color online) Transmission electron microscopy images of a sample implanted with $4.7 \times 10^{15} \mathrm{Ag}^{+}$ions $/ \mathrm{cm}^{2}$ with a kinetic energy of $3 \mathrm{keV}$ : (a) cross section showing the single plane of $\mathrm{Ag}$ NCs near the dielectric surface, (b) plan view; (c) size distribution determined from this plan view.

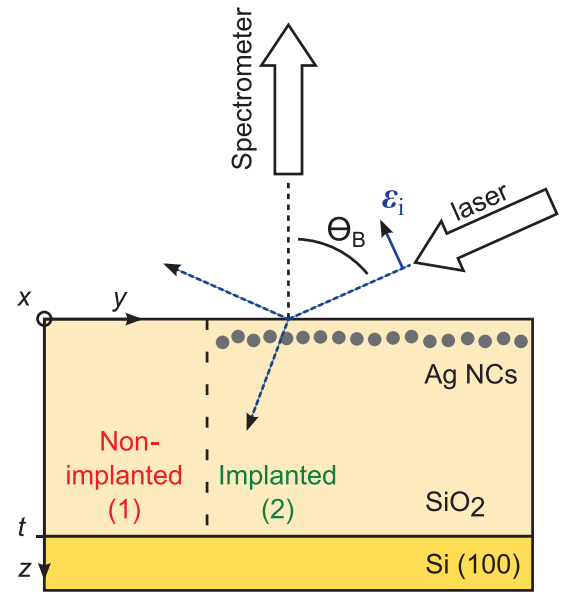

FIG. 2. (Color online) Schematic view of the experimental setup. A specific double objective geometry is used at the entrance of the spectrometer. The $p$-polarized laser light is focused under the Brewster incidence. The cross section of the sample schematizes the two-dimensional (2D) patterning obtained using the implantation process through a stencil.

\section{B. Specificities of the experimental setup}

The Ag NCs embedded near the free surface of a $\mathrm{SiO}_{2}$ layer are analyzed by Raman scattering. For isolated spherical Ag NCs embedded in silica, the LSPR is expected at $3.0 \mathrm{eV}$ [14]. Hence, using this laser excitation energy takes advantage of the plasmon resonance and results in a better Raman signal. In addition, an optical amplification can be obtained if both of the following two conditions are fulfilled: (i) the silica layer thickness corresponds to an antireflective condition and (ii) the NCs are located in the vicinity of an antinode of the electric field.

Antireflective condition. The propagation of electromagnetic waves in the $\mathrm{SiO}_{2}(\mathrm{Ag} \mathrm{NCs}) / \mathrm{Si}$ heterostructure is considered [36]. The reflectance minima of a monochromatic electromagnetic wave enlightening $\mathrm{a} \mathrm{SiO}_{2} / \mathrm{Si}$ sample under an oblique incidence (Fig. 2), occur for specific photon energies $E_{m}$. The phase condition corresponding to the successive destructive interference orders $(m=0,1,2, \ldots)$ in the silica layer writes

$$
E_{m}=\left(m+\frac{1}{2}\right) \frac{h c}{2 L \cos \theta_{r}},
$$

where $L$ is the optical path in the $\mathrm{SiO}_{2}$ layer, $h$ is the Planck constant, $c$ is the light velocity in vacuum, and $\theta_{r}$ is the refracted angle inside the silica layer.

Beyond this phase condition, the amplitude of the electromagnetic field is calculated in the stratified medium using a matrix formulation and taking into account the real and imaginary parts of the refractive index of $\mathrm{SiO}_{2}$ and $\mathrm{Si}$ [36]. Figure 3(a) displays the total electric field $\left|\mathcal{E}_{\text {tot }}\right|$ in the pure (i.e., nonimplanted) heterostructure for an incident plane wave (maximum amplitude $\left.\mathcal{E}_{\text {im }}\right)$ at normal incidence $\left(\theta_{r}=0\right)$. The energy of the incident and elastically scattered photons is chosen in coincidence with the $m=1$ interference order: $E_{1}=3.0 \mathrm{eV}$ (or $\lambda_{1}=413 \mathrm{~nm}$ in vacuum) for $t=210 \mathrm{~nm}$ and $n=1.47$ with $t$ the silica layer thickness and $n$ the 


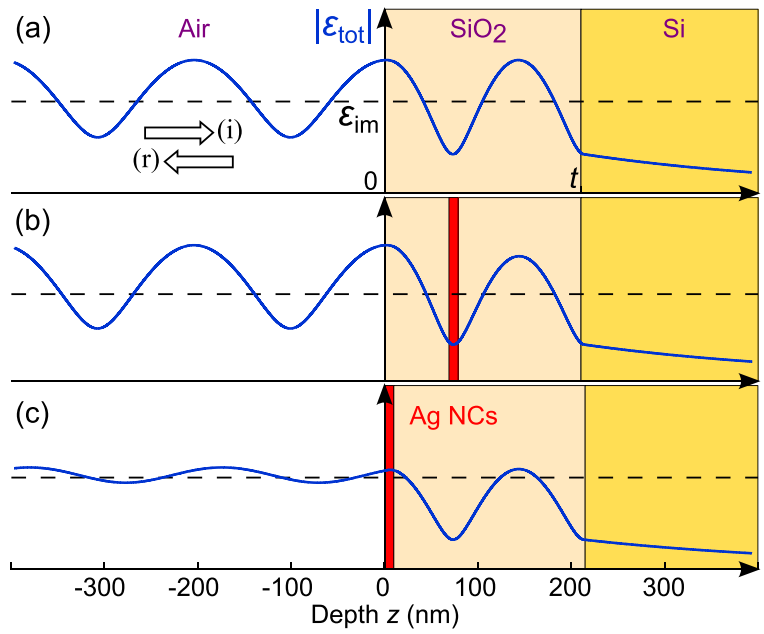

FIG. 3. (Color online) Variation vs depth of the amplitude of the total (incident $i+$ reflected $r$ ) electric field $\left|\mathcal{E}_{\text {tot }}\right|=\left|\mathcal{E}_{\mathrm{i}}+\mathcal{E}_{\mathrm{r}}\right|$ : (a) without $\mathrm{Ag} \mathrm{NCs}$, (b) with a plane of Ag NCs located at a node of the electric field into the dielectric layer, and (c) with this plane located at an antinode of the electric field near the dielectric/air interface. The wavelength in vacuum is chosen at $413 \mathrm{~nm}$ in coincidence with the plasmon frequency, the thickness of the layer is $210 \mathrm{~nm}$, the incidence is normal, the diameter of the Ag NCs is $5 \mathrm{~nm}$, and the amount of silver equals $5 \times 10^{16}$ atoms $/ \mathrm{cm}^{2}$.

(real) index of refraction. Note that the chosen silica layer thickness $t$ corresponds to $3 / 4$ of the wavelength into the layer $t=L / n \approx 3 \lambda_{1} /(4 n)$. Figure 3(a) displays the resulting stationary wave in the silica layer $(0<z<t)$ and the strong absorption in the Si substrate $(z>t)$. In air $(z<0)$, the total electric field is partially stationary because it results from the sum of the incident and reflected plane waves: $\mathcal{E}_{\text {tot }}=\mathcal{E}_{\mathrm{i}}+\mathcal{E}_{\mathrm{r}}$. Despite the antireflecting conditions, the reflectance does not totally vanish, but the amplitude of the electric field $\left|\mathcal{E}_{\text {tot }}\right|$ is rightly at maximum on the free surface of the sample $(z=0)$.

Position of the NCs. Positioning the NCs in the vicinity of the free surface $(z=0)$, will enhance the coupling between all these NCs and the electromagnetic field. Figure 3(c) reports the total electric field $\left|\mathcal{E}_{\text {tot }}\right|$ in the implanted heterostructure for an incident plane wave (maximum amplitude $\mathcal{E}_{\text {im }}$ ) at normal incidence $\left(\theta_{r}=0\right)$. The dielectric response of the composite layer is calculated by using the quasistatic approximation of the classical Maxwell-Garnett theory. In this theory the electronic confinement effect is accounted for by introducing a size-dependent surface plasmon damping [36] in the complex dielectric function for embedded silver.

In Fig. 3(c), the resonant absorption by the Ag NCs drastically modifies the electric field and particularly its stationary behavior in air: the presence of NCs strongly reduces the reflected field amplitude $\mathcal{E}_{\mathrm{rm}}$. The modification of the reflectance $\left|\mathcal{E}_{\mathrm{rm}} / \mathcal{E}_{\mathrm{im}}\right|^{2}$ has been calculated to reach $80 \%$.

Dark-field spectroscopy. Finally, in addition to the plasmon resonance and optical amplification, a specific experimental setup is used in order to limit spurious scattering on the detector originating from the huge Rayleigh line compared to the weak signals at very low-frequency shifts in RamanBrillouin spectroscopy. In that purpose, (i) we discard the usual backscattering geometry for both incident and scattered beams through a unique microscope objective, replacing it by a two objective system (Fig. 2): in the resulting dark-field geometry the specular reflected beam does not enter the spectrometer. Moreover, (ii) the incident angle is chosen near the Brewster value $\left(\theta_{B}=56^{\circ}\right.$ for the air $/ \mathrm{SiO}_{2}$ interface), thus limiting very efficiently the specular reflection by using a $p$-polarized incident laser beam (Fig. 2). Finally, (iii) the illumination of the charge-coupled device (CCD) detector of the spectrometer has been strictly limited to the spectral range of interest, by a built-in diaphragm hiding inelastic scattering signals at very low frequency, below $0.15 \mathrm{THz}$. The resonant excitation at $3.0 \mathrm{eV}$ was ensured using the 413-nm line of a krypton laser and the spectra were recorded with a T64000 Jobin-Yvon spectrometer.

\section{EXPERIMENTAL RESULTS}

Figure 4 reports the corrected resonant Raman spectrum recorded at $T=300 \mathrm{~K}$ on a specific sample containing a very large assembly of Ag NCs embedded in a thick silica layer. This sample, used here as a reference, has been obtained with a high dose of $\mathrm{Ag}^{+}$ions and high kinetic energy. The corrected intensity $I^{\text {corr }}$ is deduced by dividing the recorded one $I^{\exp }$ by the Bose statistics population factor:

$$
I^{\text {corr }}(v)=\frac{I^{\exp }}{|\bar{n}(v, T)+1|}, \quad \bar{n}=\left[\exp \left(\frac{h v}{k_{B} T}\right)-1\right]^{-1},
$$

where $k_{B}$ is the Boltzmann constant. This formula applies for either the Stokes or anti-Stokes process (providing using in the latter case $v<0$ ) and for all orders of scattering by whatever excitations. The examination of the corrected intensity $I^{\text {corr }}$ offers the opportunity to discriminate between Raman and photoluminescence processes [37]. In Fig. 4, despite fluctuations generated in the anti-Stokes region where the Bose statistics population factor vanishes at large frequency

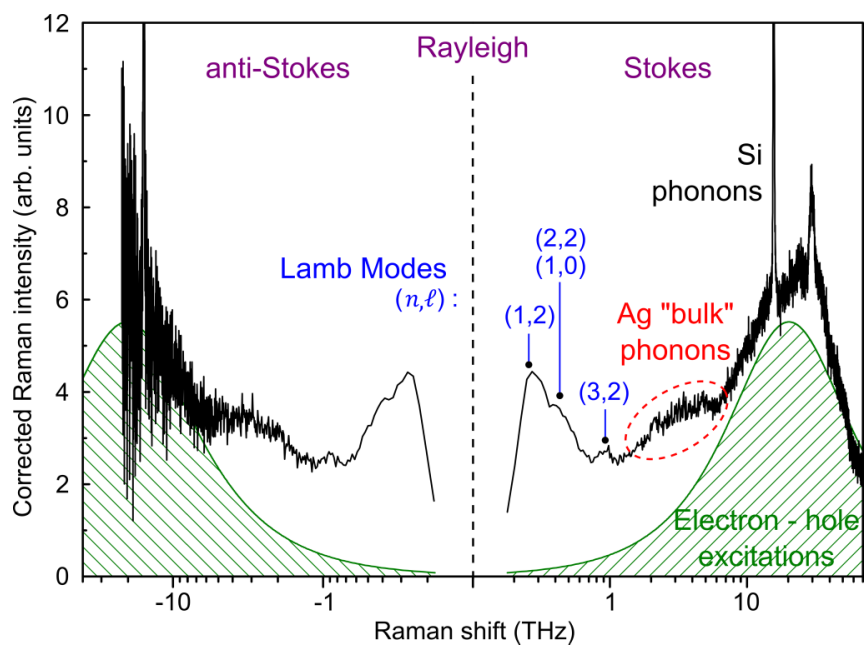

FIG. 4. (Color online) Stokes and anti-Stokes Raman spectra of a $\mathrm{SiO}_{2}(1 \mu \mathrm{m}) / \mathrm{Si}$ sample obtained by implanting $4 \times 10^{16} \mathrm{Ag}^{+}$ ions $/ \mathrm{cm}^{2}$ with a kinetic energy of $190 \mathrm{keV}$. The intensity has been corrected by the Bose population factor. The hatched area is delimited by a fit of the theoretical single-particle response. Note the unusual $\log$ scale adopted for the Raman shift. 
shift, the symmetry of the response is well verified. This demonstrates that the whole signal is due to Raman scattering events. This signal can be decomposed in three parts:

(i) The low-frequency range (below $1.2 \mathrm{THz}$ ) is dominated by the contribution of low-index Lamb modes of Ag NCs.

(ii) The high-frequency range (beyond $6 \mathrm{THz}$ ) where the first- and second-order peaks of the Si substrate emerge from a broad band which culminates near $25 \mathrm{THz}$.

(iii) A less intense and structured intermediate range (between 1.2 and $4 \mathrm{THz}$ ).

These three contributions will be now successively analyzed.

\section{A. Low-frequency range: Lamb modes}

In the low-frequency range, a well-defined and intense peak at $0.27 \mathrm{THz}$ with two shoulders near 0.5 and $0.9 \mathrm{THz}$ is clearly visible. These features are attributed to elastic deformations of the NCs (Lamb modes) corresponding to low values of the harmonic number $n$. Under resonance conditions, these modes strongly interact with the dipolar surface plasmon oscillation due to similar spatial phase modulation. They have been already widely analyzed both experimentally and theoretically $[14,15,38]$. According to the expected Raman selection rules [13] spheroidal modes can be identified in Fig. 4: the lowest frequency and most intense peak corresponds to the fundamental quadrupolar $(n=1, \ell=2)$ mode, whereas the shoulders may be attributed to its overtones ( $n=2$ and $n=$ 3 ) or to the spherical mode $(n=1, \ell=0)$. The fundamental quadrupolar mode $(n=1, \ell=2)$ of a sphere consists of a biaxial constriction of a planar section of the sphere while the orthogonal direction of the plane is expanding, and vice versa. The fundamental spherical mode $(n=1, \ell=0)$ consists of a succession of isotropic extensions/constrictions of the whole sphere. Within the continuous elastic approximation, for a metallic isotropic homogeneous sphere, a linear dependence of their frequency versus the inverse of the diameter $D$ is predicted:

$$
v_{n, \ell}=\frac{X_{n, \ell}}{D} .
$$

The elastic anisotropy of silver is expected to split the degenerate quadrupolar modes frequencies. However, the NC size distribution of our samples does not allow the observation of this effect which is hidden by the resulting inhomogeneous broadening of the Raman peaks: thus, an elastic isotropic approximation is relevant in our case. For silver NCs embedded in $\mathrm{SiO}_{2}$, the values of the parameters $X_{n, \ell}$ are deduced from previous calculations [14]. For instance one gets $X_{1,2}=$ $1.40 \mathrm{THz} / \mathrm{nm}$ for the fundamental quadrupolar mode giving a mean diameter value $D$ around $5 \mathrm{~nm}$ in agreement with TEM observations. Note that for this sample, the Raman peaks are wide testifying a rather large size dispersion.

\section{B. High-frequency range: Electron-hole excitations}

In the high-frequency range (beyond $6 \mathrm{THz}$ ), a broad band signal is clearly visible in Fig. 4. This signal is systematically present in surface-enhanced Raman scattering (SERS) spectra and generally called background. In previous works, its origin has been largely ignored and it is generally subtracted using various methods, the details of which are not reported [39]. However, quite a lot of years ago, it has been relevantly attributed to inelastic scattering by electronic excitations [34]. Uncorrelated electron-hole excitations are generated a few fs after optical excitation according to the well-recognized Landau damping mechanism following which a plasmon mode loses its coherence [11]. These excitations can thus be scattered by electronic fluctuations or vibrational excitations before their radiative recombinations lead to inelastic (Raman) processes. At each interaction step, the drastic momentum conservation rule due to translational invariance is relaxed: surface roughness and disorder at the nanoscale do play a key role for generating electron-hole excitations and SERS processes [39-42]. This wave vector breakdown can also be induced by external stimuli as adsorbed molecules or a tip, leading to an increase of the background, as recently observed $[42,43]$.

Disentangling the vibrational response and the electronhole response in Raman spectra of disordered and/or nanometer-sized systems remains difficult and is not generally performed. Only two opposite cases have been examined. On one hand, in their pioneering work [44], Shuker and Gamon analyzed Raman scattering in undoped amorphous materials leading to expressions for the first-order spectrum in terms of the VDOS: this model will be used to interpret the intermediate frequency range of the spectrum (see Sec. III C). On the other hand, Zawadsky and Cardona have developed a model [45] that accounts for the electronic contribution in "dirty" metals: the Raman scattering cross section can be calculated within the so-called collision-limited regime.

In this latter model, assuming a simple isotropic Fermi liquid, the electronic susceptibility consists of a Drude-like response, and at temperature $T$ the Stokes and anti-Stokes spectra can be written as follows:

$$
I_{e-h}(v)=I_{\max }|\bar{n}(v, T)+1| \frac{2|\nu| \tau}{1+(\nu \tau)^{2}},
$$

where the amplitude $I_{\max }$ depends on the electronic density of states at the Fermi level. The frequency-independent scattering rate $\tau^{-1}$ is attributed to impurity or phonon scattering. This model has been successfully used to account for scattering by conduction electrons in high-temperature superconductors [46].

In silver, only intraband transitions are expected under excitation in the visible range because of the large gap $(3.9 \mathrm{eV})$ for interband transitions: the electronic properties are satisfactorily described using a simple Drude approximation and a fit of the electronic response can be obtained using $\tau$ as an adjustable parameter in Eq. (4): The value of $v=\tau^{-1}$ is adjusted from the position of the maximum of the corrected intensity $I_{e-h}^{\text {corr }}=I_{e-h} /|\bar{n}(v, T)+1|: v \approx 25 \mathrm{THz}$. The fitted electron-hole excitations spectrum (delimitating a ached area) is reported in Fig. 4.

The frequency-independent scattering rate $v \approx 25 \mathrm{THz}$ is much higher than the highest phonon frequency of silver $(5.07 \mathrm{THz})$ [47] so that the electron or hole scattering processes cannot imply Ag phonons: we attribute this electron or hole scattering to static scattering by the surface of the $\mathrm{Ag}$ NCs. Indeed, $\tau=v^{-1} \approx 40 \mathrm{fs}$ is a reasonable value for the corresponding relaxation time for an electron confined in 


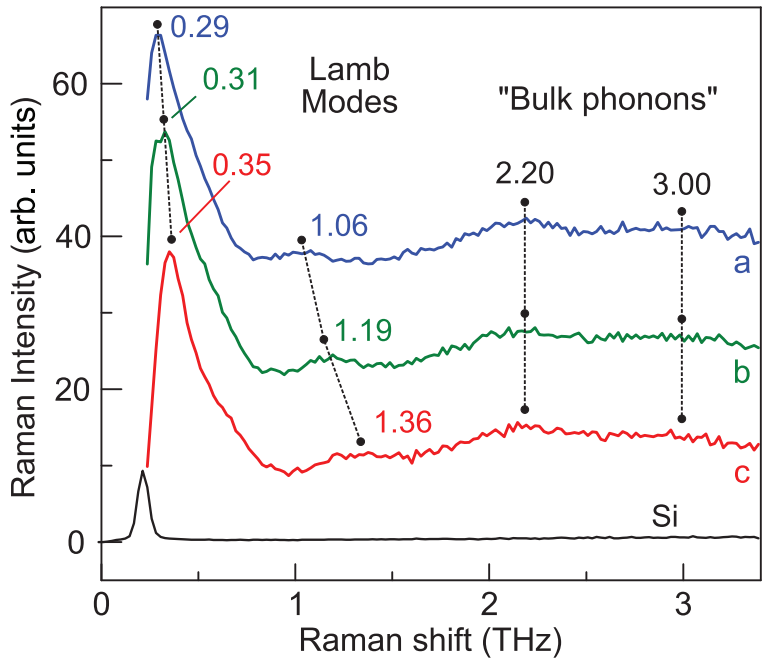

FIG. 5. (Color online) Low-frequency Raman spectra of samples obtained by implanting $\mathrm{Ag}^{+}$ions in $\mathrm{SiO}_{2}$ at low energy (3 keV) for different doses: (a) 9.4 , (b) 7.05 , and (c) $4.7 \times 10^{15}$ ions $/ \mathrm{cm}^{2}$. At the bottom, in a nonimplanted sample, only the Brillouin signal of bulk $\mathrm{Si}$ is observed. Spectra are arbitrarily shifted for clarity.

a NC with a diameter of few $\mathrm{nm}$. In agreement with this interpretation, Portales and co-workers [48] have observed that the frequency of the maximum of the electron-hole excitation spectrum varies linearly with those of the quasiconfined Lamb modes, i.e., the size of the NCs according to Eq. (3).

\section{Intermediate frequency range: "Bulk" phonons}

In order to attribute the origin of the intermediate frequency range scattering observed in Fig. 4, other implanted samples with $\mathrm{Ag}$ NCs of varying sizes have been examined. Figure 5 reports the Raman spectra of three samples implanted at very low energy $(3 \mathrm{keV})$ with different low $\mathrm{Ag}^{+}$doses. Within these conditions, only a single plane of isolated spherical Ag NCs is formed and the size distribution is better defined (Fig. 1) than in the first sample (Fig. 4). The mean diameter of the three different assemblies are deduced from the quadrupolar mode $(n=1, \ell=2)$ frequency quoted in Figs. 5(a)-5(c) and using Eq. (3): $D=4.9 \pm 0.2,4.5 \pm 0.2$, and $4.0 \pm 0.2 \mathrm{~nm}$, respectively. Figure 5 clearly evidences the frequency increase of the harmonic of the quadrupolar $(n=3, \ell=2)$ (confined) mode from 1.06 to $1.36 \mathrm{THz}$ when the dose, and consequently the mean size [35], decreases. Besides, two vibrational bands (around 2.2 and $3.0 \mathrm{THz}$ ) whose frequencies and shapes are strikingly size independent are also observed in Fig. 5. Following the work of Akemann et al. [34] performed on rough silver surfaces, we assign them to "bulk" phonons, i.e., modes whose wavelengths are generally much smaller than the NC size and consequently hardly sensitive to confinement effect.

In order to increase the ratio of the signal (scattering from a single plane of isolated $\mathrm{Ag} \mathrm{NCs}$ ) versus noise (scattering from the rest of the sample), a few hundred Raman spectra of samples for which $\mathrm{Ag}^{+}$ions are implanted through a stencil are recorded. This sample contains defined zones with (1) or without (2) NCs (see Fig. 2). Figure 6 reports the average

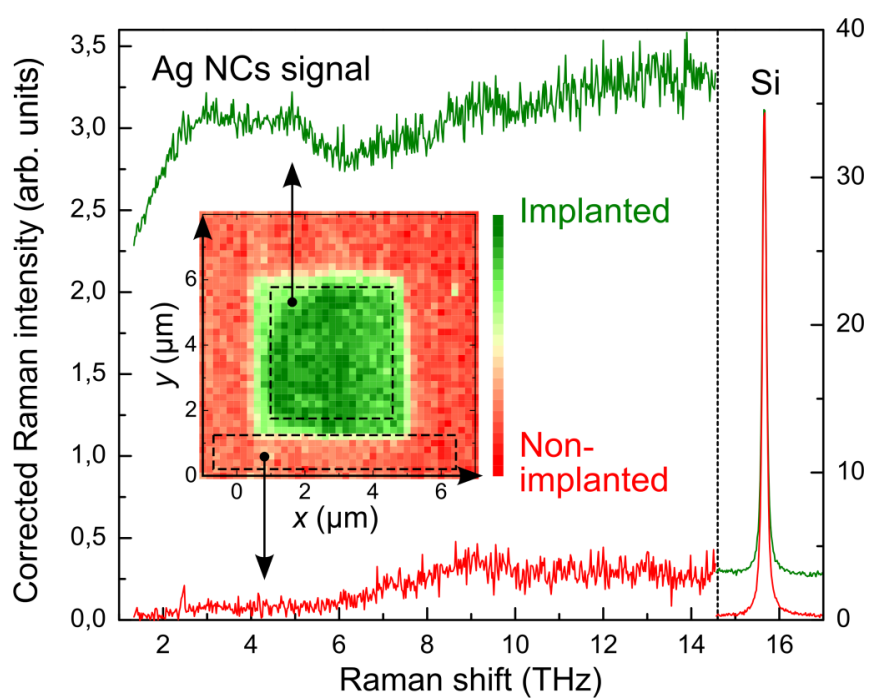

FIG. 6. (Color online) $X-Y$ scan of an $\mathrm{Ag}^{+}$implanted sample through a square hole $\left(5 \times 5 \mu \mathrm{m}^{2}\right)$ of a stencil; the linear color scale, from red to green, refers to the Raman signal intensity in the 1.5-6 THz window. On top (in green) spectrum obtained by averaging records over a squared implanted zone; at the bottom (in red) spectrum obtained by averaging over a rectangular nonimplanted zone. The spectra are normalized using the first-order Raman line of the $\mathrm{Si}$ substrate (near $16 \mathrm{THz}$ ) as a reference.

spectra of zone (1) and zone (2). The low-frequency part of the spectrum $(v<1.5 \mathrm{THz})$ corresponding to the Lamb modes is not reported here because the mask used in order to hide the Rayleigh line also hides these modes. The signal assigned to the $\mathrm{Ag}$ NCs is obtained by subtracting these two spectra. In addition, the electronic contribution $I_{e-h}$ is subtracted by describing it using Eq. (4) (see Fig. 4).

Figure 7(a) finally reports the corrected spectrum $I^{\text {corr }}$ $I_{e-h}^{\text {corr }}$ after all these operations. This spectrum precisely corresponds to the intermediate-range frequency part of the

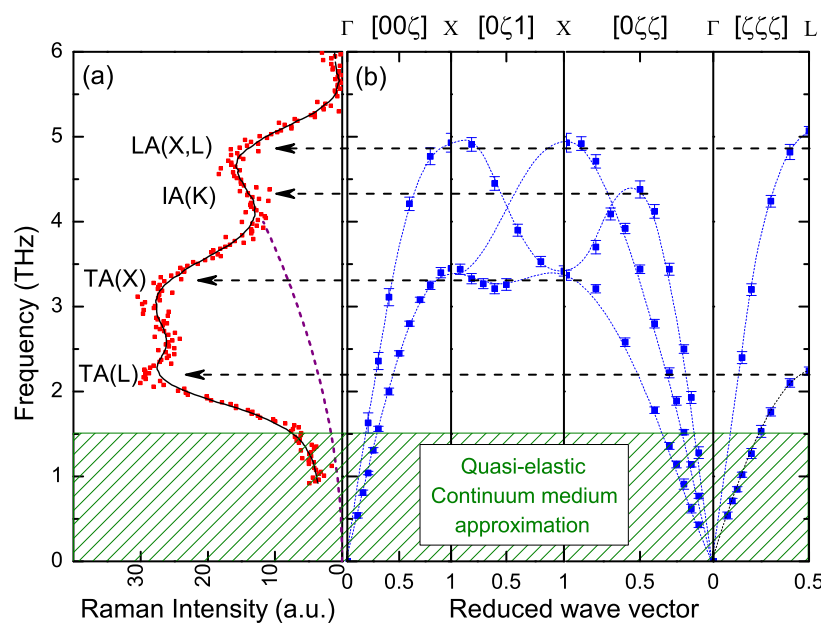

FIG. 7. (Color online) (a) Corrected Raman spectrum recorded in this work. (b) Phonon dispersion curves in bulk Ag: the dots and errors bars are the experimental data obtained by inelastic neutron scattering [47]. The arrows indicate phonon frequencies at high-symmetry points of the Brillouin zone. 
signal previously pointed out in Fig. 4. Figure 7(b) reports the experimental phonon dispersion curves determined from neutron inelastic scattering in bulk Ag [47]. The corrected Raman spectrum displayed in Fig. 7(a) is very similar to the VDOS that one could deduce from these phonon dispersion curves: the maxima and cutoff in the corrected Raman spectrum correspond to Van Hove singularities of the VDOS at critical points $L, X$, and $K$ of the Brillouin zone [49].

We claim that this corrected Raman spectrum represents with a good approximation the VDOS of the NCs. The attribution of the spectrum of Fig. 7(a) to the NCs VDOS is here explained using a classical description where the Raman-scattering spectral response is described as the spacetime Fourier component of the autocorrelation function of the dielectric tensor fluctuations. Following Shuker and Gamon, the Raman spectra depends on the frequency $v$ as [44]

$$
I_{\alpha}(v)=\sum_{j} C_{j, \alpha} V_{j} \frac{1}{v_{j}}\left[\bar{n}\left(v_{j}, T\right)+1\right] \delta\left(|v|-v_{j}\right) .
$$

This expression refers to a given experimental setup geometry, simply labeled $\alpha$. The summation in Eq. (5) runs over all the $j$ modes whose eigenfrequency is $v_{j}=|v| . V_{j}$ is the scattering volume, i.e., the volume in which the atoms displacements are spatially correlated for each mode $j$. The coupling factor $C_{j, \alpha}\left(v_{j}\right)$ depends on the mode symmetry, optical properties of the sample, electronic properties of the scatter unit, and electron-phonon couplings. In a Ag NC whose size is much smaller than the wavelength of the light and much larger than the atomic spacing, $V_{j}$ is with a good approximation the volume of this NC (i.e., of the confined and polarizable electron gas) for all the modes. In addition, for all the $\mathrm{Ag}$ NCs investigated under the same geometry, the optical and electronic properties are the same for all modes. This is due to the specificity of the sample and the very small energy range implied. For Ag NCs, neither privileged orientation nor specific electronic transition in the visible range is present. As a result, the subscript $\alpha$ can be omitted. If the few Lamb modes are excluded, the discrete sum in Eq. (5) can be replaced by the VDOS for each vibrational band (b):

$$
I(v)=\sum_{b} C_{b}(|v|) \frac{1}{v}[n(v, T)+1] g_{b}(|v|) .
$$

Phenomenological spatial correlation models [37,44,50,51] have been proposed to account for the coupling factor $C_{b}(|v|)$. In silver, with one atom per unit cell, all the modes belong to acoustical branches and only the deformation potential mechanism is relevant for describing electron-phonon interactions [38]. Assuming that this mechanism does not depend on the polarization of the modes (longitudinal LA or transverse TA), one can write

$$
I(v) \propto C(|v|) \frac{1}{v}[\bar{n}(v, T)+1] g(|v|) .
$$

By making now the crude approximation that $C(|\nu| /|\nu|)$ is not frequency dependent, the twofold degenerated TA contribution should be twice the LA one. Indeed, the area under the dotted line in Fig. 7(b) shows that the LA contribution can be estimated as one-third of the total integrated VDOS. The weak frequency dependence of $C(|\nu|) /|\nu|$ has already been verified in disordered covalent semiconductors [37,51,52] and in vitreous materials [44]. In particular the same approximation has been very recently used to account for thermal properties in densified glasses [53]. One finally gets

$$
\left[I^{\text {corr }}-I_{e-h}^{\text {corr }}\right](v) \propto g(|v|)=\operatorname{VDOS}
$$

for both Stokes $(v>0)$ and anti-Stokes $(v<0)$ Raman scattering. As a result of this discussion, the spectrum reported in Fig. 7(b) represents with a good approximation the VDOS of the NCs.

\section{DISCUSSION}

\section{A. Comparison with theoretical VDOS}

The experimental VDOS is now compared with theoretical predictions. Atomistic simulations using a silver tight-binding potential [54] has been performed. The phonon modes are obtained from the calculation and diagonalization of the dynamical matrix. The bulk VDOS is generated from an isotropic sampling of the Brillouin zone using a periodic boundary cell. Spherical NCs are designed with atoms initially placed on a perfect cubic close-packed crystal structure using the bulk cell parameters of the modeled material [24]. The potential energy is then relaxed using a conjugated gradient algorithm. This procedure ensures that all vibration eigenfrequencies are real. Our model however omits the possible existence of surface reconstructions and dangling bonds. Two limiting cases for the boundary conditions are considered: the "free" boundary conditions, corresponding to a free nanoparticle in vacuum, and the "rigid" boundary conditions, where the surface atoms are fixed, a situation which corresponds to a nanoparticle embedded in a rigid matrix.

Figure 8(a) reports the VDOS of bulk silver calculated by atomistic calculations and the experimental one for an assembly of NCs with $D \approx 4 \mathrm{~nm}$ : both curves present strong similarities. The agreement between experiment and theory is good for the lowest frequency modes $(<1.5 \mathrm{THz})$ : the experimental VDOS follows the $v^{2}$ Debye law expected for 3D systems, attesting that these modes are well described within the continuous elastic medium approximation [16,24]. At higher frequencies, some discrepancies are observed:

(i) On the high-frequency part of the TA band, between 1.5 and $2.5 \mathrm{THz}$, an excess of modes is clearly observed in the VDOS of NCs.

(ii) In the highest frequency range, beyond $4 \mathrm{THz}$, corresponding to LA modes near Brillouin-zone edges (see Fig. 7), a strong softening and an overall shift are observed with a high-frequency tail beyond the cutoff of the bulk phonons, near $5 \mathrm{THz}$.

The present work thus experimentally confirms the frequency redistribution of modes, already theoretically predicted by various authors [19-21] who suggested the following explanations:

(i) The so-called "high-frequency tail" was generally attributed to a stiffening of the chemical bonding between inner atoms. The global shrinkage of their nearest-neighbor distances is due to the contraction of lower coordinated atoms at the surface [21]. In multigrain systems, the anomaly was attributed to grain-boundary atoms [31] but it is not relevant here because the NCs are well isolated from each other. 


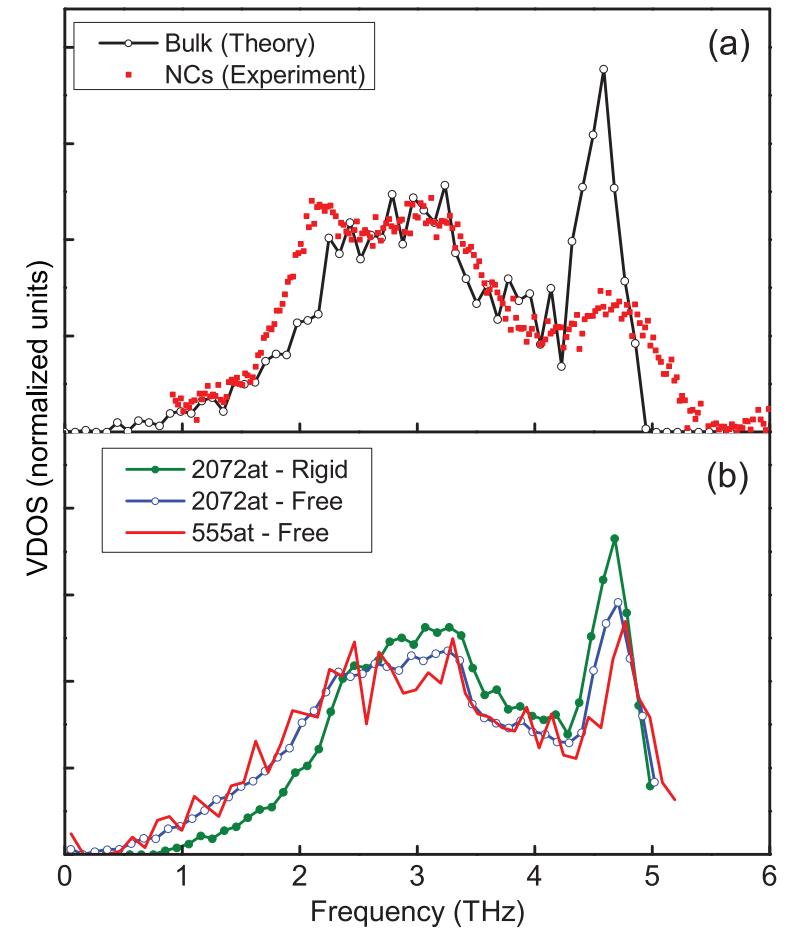

FIG. 8. (Color online) (a) Comparison of VDOS calculated for bulk Ag using atomic-scale simulations (continuous black line) and for an assembly of $\mathrm{Ag}$ NCs (mean size $D \approx 4 \mathrm{~nm}$ ) obtained experimentally (red dots); (b) Calculated VDOS of a spherical NC with 2072 atoms $\mathrm{Ag}$ atoms $(D=4 \mathrm{~nm})$ for free and rigid boundary conditions, and of a NC with 555 atoms. All the integrated areas have been normalized.

(ii) The "low-frequency excess" was attributed to vibrations of undercoordinated surface atoms characterized by a different binding. The enhancement of the VDOS at very low frequency (Debye regime) could then be explained by a reduction of dimensionality leading to a change on frequency dependence from quadratic ( $3 \mathrm{D}$ for volume modes) to linear ( $2 \mathrm{D}$ for surface modes).

\section{B. Size, strain, and disorder effects}

In order to analyze more precisely these effects, we have on one hand calculated the VDOS of a 4-nm-diameter spherical Ag NC for two opposite boundary conditions, free or rigid, and on the other hand checked experimentally the strain field in isolated NCs.

Figure 8(b) reports the VDOS of a NC with 2072 atoms Ag atoms $(D=4 \mathrm{~nm})$ for both free and rigid boundary conditions. The low-frequency excess (i) and the high-frequency tail (ii) are clearly visible in both cases but they are more pronounced in the free boundary conditions case, demonstrating that displacements of Ag surface atoms play a significant role. As a matter of fact one observes in Fig. 8(b) that increasing the surface-to-volume ratio by reducing the size of the $\mathrm{NC}$ to 555 atoms $(D=2.65 \mathrm{~nm})$ does enhance both low- and high-frequency anomalies.

The frequency redistribution of vibrational modes in $\mathrm{NCs}$ is at the origin of the modification of thermodynamic properties [19-21], namely the lattice specific heat $C_{v}[22]$ :

$$
C_{v}=k_{B} \int_{0}^{\infty} g(v)\left(\frac{h v}{k_{B} T}\right)^{2} \frac{e^{h v / k_{B} T}}{\left(e^{h v / k_{B} T}-1\right)^{2}} d v .
$$

The Debye approximation consists of using in Eq. (9), for $0 \leqslant$ $v \leqslant k_{B} T_{D} / h$, the following expression of the VDOS, $g_{D}(v)=$ $18 \pi N_{a}\left(h / k_{B} T_{D}\right)^{3} v^{2}$, where $T_{D}$ is the Debye temperature. Using Eq. (9) and the bulk and NC VDOS reported in Fig. 8, an effective Debye temperature is determined by fitting the calculated $C_{v}(T)$ curves with the Debye function $C_{v, D}(T)$. In this Debye function, we used the expression of $g_{D}(v)$ with $T_{D}$ as the only adjustable parameter. From the atomic simulation of bulk Ag, one finds $T_{D}=210 \pm 3 \mathrm{~K}$ in good agreement with the experiment $(215 \mathrm{~K})$ [22]. Simulations of NCs with free boundaries conditions yield $T_{D}=205 \mathrm{~K}$. The experimental VDOS extracted from the Raman spectrum can be also used in Eq. (9) to analyze the thermal behavior [53]. In our case, the best fit is obtained with $T_{D}=195 \mathrm{~K}$ : this gives a confirmation of the lowering of $T_{D}$ with the size reduction, as predicted theoretically [20].

Internal strain effects have been already examined experimentally using simulations to account for vibrational frequency shifts in NCs $[28,55,56]$. Because strain inhomogeneity may induce broadening, we have performed strain mapping of Ag NCs by analyzing their high-resolution electron microscopy (HREM) images using the geometrical phase analysis (GPA) technique [57]. This technique has been widely used for strain measurements in epitaxial layers or MOS devices [58] but only a few studies concern strain in small nanocrystals [59]. The GPA technique consists of decomposing a HREM image in the Fourier space and analyzing the Fourier components of two spatial frequencies corresponding to two systems of periodic fringes in the direct space. From the geometric phase variations of noncollinear Fourier components, the local changes of periodicity are deduced by comparison with an unstrained reference zone. The spatial analysis of these periodicity variations provides the mapping of strains, rotations, and shears of the strained structure in the observation plane. The GPA technique measures these phase shifts and maps the strain in "small" crystalline zones (within few tens of $\mathrm{nm}$ ) with respect to a region taken as a reference (i.e., unstrained) with accuracy of $0.1 \%$ and subnanometric spatial resolution. Cross-sectional and plan-view specimens were prepared for TEM examination by the tripod method and finished by a gentle ion milling step. A FEI Tecnai microscope equipped with a field emission gun and a spherical aberration corrector and operating at $200 \mathrm{keV}$ was used for imaging.

The strain field inside an embedded nanocrystal (see Fig. 9) is found to be homogeneous (standard deviation less than $0.1 \%$ ). The same conclusion is confirmed by examining several NCs. However the spatial resolution of the GPA technique remains around $0.7 \mathrm{~nm}$ for the images shown in Fig. 9 and thus surface effects at the atomic scale cannot be analyzed. Absolute measurements of this homogeneous strain would have required a time-consuming statistical study and the use of an outside reference (such as the Si substrate). We simply refer here to already published data by Hofmeister and co-workers [60,61] on assemblies of Ag NCs obtained by implantation in $\mathrm{SiO}_{2}$. 

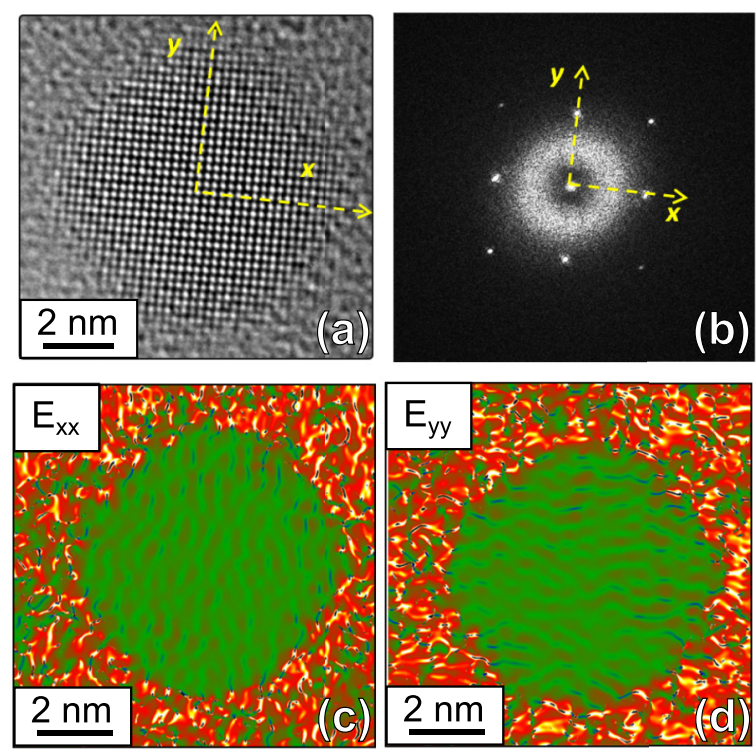

FIG. 9. (Color online) (a) High-resolution electron microscopy image of a well-oriented $\mathrm{Ag} \mathrm{NC}$ embedded in $\mathrm{SiO}_{2}$. (b) Corresponding diffraction pattern showing the cubic structure of the $\mathrm{Ag} \mathrm{NC}$. (c),(d) Maps of strain field components obtained using the geometric phase analysis method where the reference is taken at the center of the NC.

Using HREM and extended x-ray-absorption fine-structure measurements, an interface stress has been evaluated as $f \approx 1.3 \pm 0.9 \mathrm{~N} / \mathrm{m}$ [61]. The relative change of the lattice parameter in a particle of diameter $D$ is given by [62]

$$
\frac{\Delta a}{a}=-\frac{4}{3} \frac{f}{B D}
$$

Using the bulk modulus value of silver $B=100.7 \mathrm{GPa}$ [22] and a mean diameter $D=4 \mathrm{~nm}$ one deduces a compressive strain $\Delta a / a \approx-0.43 \%$. Since the Grüneisen parameters in $\mathrm{Ag}$ have been estimated as $\gamma \approx 2.5$ [63], the expected relative frequency shift is $\Delta v / v=-3 \gamma \Delta a / a \approx 3.2 \%$.

A homogeneous strain in each $\mathrm{NC}$, as shown here by the GPA technique, with an absolute value $\Delta a / a \approx-0.43 \%$ would induce a shift around $0.16 \mathrm{THz}$ for its higher frequency part of the VDOS in good agreement with the blueshift observed experimentally for the LA band in Fig. 8(b). For TA modes, a lower blueshift is expected and can therefore not explain the frequency enhancement of the VDOS at low frequencies around $2 \mathrm{THz}$. Moreover the broadening of the LA band is still lower in simulated spectra than in experiment.
The larger broadening of the experimental VDOS can be explained by the damping of all the modes due to internal disorder and anisotropy effects (homogeneous broadening). This broadening is amplified by the dispersion of sizes and shapes (inhomogeneous broadening). In that sense, of particular relevance are the calculations performed for various morphologies on vibrational modes in Ag NCs [23]. In these calculations, a large softening of the high-frequency modes and a low-frequency excess have been found for some specific morphologies, giving a support for this shape-distribution effect. Finally it is important to note that none of the atomistic models includes explicitly the embedding matrix which may play an important role.

\section{CONCLUSION}

In this work, an experimental investigation of the spectral response in the $\mathrm{THz}$ regime of nanocrystals has been presented. By exploiting simultaneously optical amplification, plasmon resonance, and dark-field geometry for low-frequency Raman scattering and imaging, accurate signals have been obtained from a single plane of Ag NCs. Their dynamical response has been particularly analyzed for electronic excitations and bulk or surface atomic vibrations. On one hand, the continuous and broad spectrum (generally observed in SERS spectra) has been well accounted for as inelastic scattering by electron-hole excitations. On the other hand, a reliable picture of the VDOS has been obtained, thus offering an opportunity to validate theoretical predictions deduced from atomic-scale calculations. The occurrence of a high-frequency tail beyond the bulk cutoff has been confirmed, thus validating the hypothesis of an internal strain effect on inner atoms. The homogeneity of the strain field in a single nanocrystal has been originally examined using GPA coupled with HREM. This work gives insights concerning the limit of elastic continuous media approximation, and the crucial role of surface modes. It offers opportunities to assess thermodynamics properties at the nanoscale. However, the dynamical response of embedded nanocrystals assemblies is highly affected by disorder, size distribution, anisotropy, and boundary effects. In that sense, much effort has to be invested to obtain well-controlled assemblies of NCs to get more accurate information at this scale.

\section{ACKNOWLEDGMENTS}

M.B. thanks the University of Toulouse and Région Midi-Pyrénées for financial support. Part of this work was supported by Programme Investissements d'Avenir under the program ANR-11-IDEX-0002-02, reference ANR-10-LABX0037-NEXT.
[1] G. V. Hartland, Chem. Rev. 111, 3858 (2011).

[2] S. Nie, Science 275, 1102 (1997).

[3] G. Baffou and R. Quidant, Laser Photon. Rev. 7, 171 (2013).

[4] H. A. Atwater and A. Polman, Nat. Mater. 9, 205 (2010).
[5] S. Linic, P. Christopher, and D. B. Ingram, Nat. Mater. 10, 911 (2011).

[6] S. C. Warren, D. A. Walker, and B. A. Grzybowski, Langmuir 28, 9093 (2012).

[7] P. Spinelli and A. Polman, Opt. Express 20, A641 (2012). 
[8] X. Zhang, Y. L. Chen, R.-S. Liu, and D. P. Tsai, Rep. Prog. Phys. 76, 046401 (2013).

[9] G. Prieto, J. Zečević, H. Friedrich, K. P. de Jong, and P. E. de Jongh, Nat. Mater. 12, 34 (2013).

[10] S. Mukherjee, F. Libisch, N. Large, O. Neumann, L. V. Brown, J. Cheng, J. B. Lassiter, E. A. Carter, P. Nordlander, and N. J. Halas, Nano Lett. 13, 240 (2013).

[11] D. Steinmüller-Nethl, R. A. Höpfel, E. Gornik, A. Leitner, and F. R. Aussenegg, Phys. Rev. Lett. 68, 389 (1992).

[12] A. Arbouet, C. Voisin, D. Christofilos, P. Langot, N. Del Fatti, F. Vallée, J. Lermé, G. Celep, E. Cottancin, M. Gaudry, M. Pellarin, M. Broyer, M. Maillard, M. P. Pileni, and M. Treguer, Phys. Rev. Lett. 90, 177401 (2003).

[13] E. Duval, A. Boukenter, and B. Champagnon, Phys. Rev. Lett. 56, 2052 (1986).

[14] B. Palpant, L. Saviot, J. Lermé, B. Prével, M. Pellarin, E. Duval, A. Perez, and M. Broyer, Eur. Phys. J. D 9, 585 (1999).

[15] L. Saviot, N. Combe, and A. Mlayah, Phys. Rev. B 85, 075405 (2012).

[16] V. Juvé, A. Crut, P. Maioli, M. Pellarin, M. Broyer, N. Del Fatti, and F. Vallée, Nano Lett. 10, 1853 (2010).

[17] H. E. Sauceda, D. Mongin, P. Maioli, A. Crut, M. Pellarin, N. D. Fatti, F. Vallée, and I. L. Garzón, J. Phys. Chem. C 116, 25147 (2012).

[18] H. Lamb, Proc. London Math. Soc. s1-13, 189 (1882).

[19] R. Meyer, L. J. Lewis, S. Prakash, and P. Entel, Phys. Rev. B 68, 104303 (2003).

[20] B. Roldan Cuenya, L. K. Ono, J. R. Croy, K. Paredis, A. Kara, H. Heinrich, J. Zhao, E. E. Alp, A. T. DelaRiva, A. Datye, E. A. Stach, and W. Keune, Phys. Rev. B 86, 165406 (2012).

[21] A. Kara and T. S. Rahman, Phys. Rev. Lett. 81, 1453 (1998).

[22] C. Kittel, Introduction to Solid State Physics (Wiley, New York, 2004), p. 704.

[23] G. A. Narvaez, J. Kim, and J. W. Wilkins, Phys. Rev. B 72, 155411 (2005).

[24] N. Combe and L. Saviot, Phys. Rev. B 80, 035411 (2009).

[25] Q. Bian, S. Bose, and R. Shukla, J. Phys. Chem. Solids 69, 168 (2008).

[26] P. M. Derlet, R. Meyer, L. J. Lewis, U. Stuhr, and H. Van Swygenhoven, Phys. Rev. Lett. 87, 205501 (2001).

[27] D. Y. Sun, X. G. Gong, and X.-Q. Wang, Phys. Rev. B 63, 193412 (2001).

[28] P. Armstrong and W. Peukert, J. Nanopart. Res. 14, 1288 (2012).

[29] N. Combe, P.-M. Chassaing, and F. Demangeot, Phys. Rev. B 79, 045408 (2009).

[30] N. N. Ovsyuk and V. N. Novikov, Phys. Rev. B 53, 3113 (1996).

[31] P. M. Derlet and H. Van Swygenhoven, Phys. Rev. Lett. 92, 035505 (2004).

[32] U. Stuhr, H. Wipf, K. H. Andersen, and H. Hahn, Phys. Rev. Lett. 81, 1449 (1998).

[33] B. Fultz, C. C. Ahn, E. E. Alp, W. Sturhahn, and T. S. Toellner, Phys. Rev. Lett. 79, 937 (1997).

[34] W. Akemann, A. Otto, and H. R. Schober, Phys. Rev. Lett. 79, 5050 (1997).
[35] R. Carles, C. Farcau, C. Bonafos, G. Benassayag, B. Pécassou, and A. Zwick, Nanotechnology 20, 355305 (2009).

[36] R. Carles, C. Farcau, C. Bonafos, G. Benassayag, M. Bayle, P. Benzo, J. Groenen, and A. Zwick, ACS Nano 5, 8774 (2011).

[37] A. Zwick and R. Carles, Phys. Rev. B 48, 6024 (1993).

[38] G. Bachelier and A. Mlayah, Phys. Rev. B 69, 205408 (2004).

[39] J. T. Hugall, J. J. Baumberg, and S. Mahajan, J. Phys. Chem. C 116, 6184 (2012).

[40] A. Otto and M. Futamata, Top. Appl. Phys. 103, 147 (2006).

[41] A. Otto, W. Akemann, and A. Pucci, Isr. J. Chem. 46, 307 (2006).

[42] C. Farcau and S. Astilean, Chem. Commun. 47, 3861 (2011).

[43] S. Mahajan, R. M. Cole, J. D. Speed, S. H. Pelfrey, A. E. Russell, P. N. Bartlett, S. M. Barnett, and J. J. Baumberg, J. Phys. Chem. C 114, 7242 (2010).

[44] R. Shuker and R. Gammon, Phys. Rev. Lett. 25, 222 (1970).

[45] A. Zawadowski and M. Cardona, Phys. Rev. B 42, 10732 (1990).

[46] D. Reznik, M. V. Klein, W. C. Lee, D. M. Ginsberg, and S.-W. Cheong, Phys. Rev. B 46, 11725 (1992).

[47] W. Kamitakahara and B. Brockhouse, Phys. Lett. A 29, 639 (1969).

[48] H. Portales, E. Duval, L. Saviot, M. Fujii, M. Sumitomo, and S. Hayashi, Phys. Rev. B 63, 233402 (2001).

[49] R. Carles, N. Saint-Cricq, J. B. Renucci, M. A. Renucci, and A. Zwick, Phys. Rev. B 22, 4804 (1980).

[50] H. Richter, Z. Wang, and L. Ley, Solid State Commun. 39, 625 (1981).

[51] I. Campbell and P. Fauchet, Solid State Commun. 58, 739 (1986).

[52] R. Carles, A. Zwick, M. Renucci, and J. Renucci, Solid State Commun. 41, 557 (1982).

[53] G. Carini, G. Carini, G. D’Angelo, G. Tripodo, G. Di Marco, C. Vasi, and E. Gilioli, Phys. Rev. Lett. 111, 245502 (2013).

[54] F. Cleri and V. Rosato, Phys. Rev. B 48, 22 (1993).

[55] P. S. Pizani, A. Mlayah, J. Groenen, R. Carles, and A. Claverie, Appl. Phys. Lett. 66, 1927 (1995).

[56] C. Q. Sun, L. K. Pan, C. M. Li, and S. Li, Phys. Rev. B 72, 134301 (2005).

[57] M. Hÿtch, E. Snoeck, and R. Kilaas, Ultramicroscopy 74, 131 (1998).

[58] M. Hÿtch, F. Houdellier, F. Hüe, and E. Snoeck, Nature (London) 453, 1086 (2008).

[59] C. L. Johnson, E. Snoeck, M. Ezcurdia, B. Rodríguez-González, I. Pastoriza-Santos, L. M. Liz-Marzán, and M. J. Hÿtch, Nat. Mater. 7, 120 (2007).

[60] W. Cai, H. Hofmeister, and M. Dubiel, Eur. Phys. J. D 13, 245 (2001).

[61] M. Dubiel, H. Hofmeister, E. Schurig, E. Wendler, and W. Wesch, Nucl. Instr. Methods Phys. Res. B 166-167, 871 (2000).

[62] J. Vermaak, C. Mays, and D. Kuhlmann-Wilsdorf, Surf. Sci. 12, 128 (1968).

[63] V. P. Singh and M. P. Hemkar, J. Phys. F: Met. Phys. 7, 761 (1977). 